\title{
FABRICAÇÃO DE SENSOR ORGÂNICO FLEXÍVEL PARA APLICAÇÃO EM TERAPIA COM LUZ AZUL
}

Mariana de Melo Silva ${ }^{\prime}$ Giovana Ribeiro Ferreira ${ }^{2}$

Thiago Schimitberger ${ }^{3}$

Rodrigo Fernando Bianchi ${ }^{4}$

\section{Resumo}

A fototerapia com luz azul $(460 \mathrm{~nm}-490 \mathrm{~nm})$ é o procedimento mais utilizado para o tratamento da icterícia em recém-nascidos. Contudo, dois fatores alteraram drasticamente a eficiência desse tratamento: $(I) \circ$ espectro da fonte luminosa e (2) a dose de radiação absorvida pelos neonatos. Este trabalho apresenta o desenvolvimento de detectores de radiação orgânicos e flexíveis, de baixo custo, de bom desempenho e de fácil operação para monitoramento da radiação incidente em neonatos ictéricos sob tratamento fototerápico. O princípio de funcionamento dos dispositivos baseia-se na resposta óptica de filmes finos à base de dois materiais orgânicos luminescentes: o tris (8-hidroxiquinolinato de alumínio) - $\mathrm{Alq}_{3}$ e o poli[2-metóxi-5-(2'-etilóxi)-p-fenilenovinileno] - MEH-PPV, dispersos em matriz de poliestireno - PS sob efeito de luz azul. Os resultados obtidos mostram que a radiação atua no sentido de alterar a intensidade de máxima emissão do vermelho ao verde, passando pelo amarelo, dos filmes de PS/MEH-PPV/Alq ${ }_{3}$. Esse resultado é atribuído à fotodegradação do $\mathrm{MEH}-\mathrm{PPV}$ e, consequentemente, as mudanças na sobreposição entre os espectros de emissão do $\mathrm{Alq}_{3}$ e de absorção desse polímero. A resposta óptica dos filmes com a radiação foi usada para fabricar um sensor orgânico flexível, inédito e de custo inferior a $R \$ 0,50$, para controle das condições de exposição de neonatos ictéricos à fototerapia com luz azul. Palavras-chave: Eletrônica orgânica; Nanotecnologia; Dispositivos; Fototerapia.

\section{FABRICATION OF ORGANIC AND FLEXIBLE SENSOR FOR APLICATION IN BLUE-LIGHT THERAPY}

\begin{abstract}
Blue-light therapy $(460 \mathrm{~nm}-490 \mathrm{~nm})$ is the most common treatment of jaundice in newborns. However, the efficiency of this treatment is dependent of two factors: ( $I$ ) the spectrum of light source and (2) the delivered dose to the neonates. This work presents the development of a low-cost, flexible, good performance and an easy detector operation based on organic materials for evaluation of radiation dose delivered by the phototherapy units to jaundice newborns. The operation principles of the device is based on the optical response of poly [2-methoxy-5-(2'-ethylhexyloxy)-pphenylene - MEH-PPV, tris (8-hydroxyquinolinato aluminum)-Alq $q_{3}$ and polystyrene-PS thin films under the effects of blue-light. It is observed that the optical response of the PS/MEH-PPV/Alq films changes from red to yellow, and then to green with the radiation exposure. It is attributed to the photodegradation process of MEH-PPV and also to the spectral overlap between the photoemission of $\mathrm{Alq}_{3}$ and the absorption of MEH-PPV. The optical response of PS/MEH-PPV/Alq films was used to develop a novel organic flexible and low cost $(<$ US\$ 0.25) smart sensor to ensure the effectiveness of phototherapy treatment.
\end{abstract}

Key words: Organic electronics; Nanotecnology; Devices; Phototherapy.

\footnotetext{
' Química. Universidade Federal de Ouro Preto - UFOP, Instituto de Ciências Exatas e Biológicas, Departamento de Física, Campus Universitário, Morro do Cruzeiro, Cep 35400-000, Ouro Preto, MG, Brasil. E-mail: marianameloquimica@gmail.com

${ }^{2}$ Mestre em Engenharia de Materiais - Redemat, Universidade Federal de Ouro Preto - UFOP, Instituto de Ciências Exatas e Biológicas, Departamento de Física, Campus Universitário, Morro do Cruzeiro, Cep 35400-000, Ouro Preto, MG, Brasil.

E-mail: giovanaribeiro_ufop@yahoo.com.br

${ }^{3}$ Físico. Universidade Federal de Ouro Preto - UFOP, Instituto de Ciências Exatas e Biológicas, Departamento de Física,

Campus Universitário, Morro do Cruzeiro, Cep 35400-000, Ouro Preto, MG, Brasil. E-mail: tschimit@gmail.com

${ }^{4}$ Doutor em Ciências e Engenharia de Materiais. Professor Dr. Universidade Federal de Ouro Preto - UFOP, Instituto de Ciências Exatas e Biológicas, Departamento de Física, Campus Universitário, Morro do Cruzeiro, Cep 35400-000, Ouro Preto, MG, Brasil. E-mail: bianchi@iceb.ufop.br
} 


\section{INTRODUÇÃO}

A hiperbilirrubinemia ou icterícia é uma das patologias neonatais que têm recebido grande atenção nos últimos anos. (') Segundo a Sociedade Brasileira de Pediatria, ${ }^{(2)}$ apenas no Brasil, por ano, cerca de I,5 milhão de recém-nascidos apresenta icterícia já nos seus primeiros dias de vida e, destes, cerca de 250 mil em estado grave com risco de neurotoxidade, kernicterus ou óbito. ${ }^{(3)} \mathrm{A}$ icterícia é caracterizada pela alta concentração de bilirrubina no sangue, ${ }^{(4)}$ sendo a fototerapia com luz azul (460 nm - $490 \mathrm{~nm}$ ) o procedimento mais utilizado para o tratamento dessa patologia, por ser de simples aplicação e por diminuir de forma eficaz os níveis de bilirrubina no sangue do neonato. Entretanto, vários autores têm alertado sobre o uso de sistemas de iluminação inadequados e sobre o mau posicionamento dos recém-nascidos frente à fonte de luz. Esses fatores diminuem a eficiência da dose de radiação azul absorvida por neonatos. Por exemplo, toalhas e fraldas de tamanho excessivo que comumente são colocadas entre a fonte de radiação e o recém-nascido funcionando, portanto, como elementos de obstrução à passagem da radiação. Não obstante aos problemas causados pelo mau uso da fototerapia no tratamento da icterícia, essa doença está diretamente relacionada à má formação hepática dos recém-nascidos. Nesse contexto, têm-se observado na literatura a correlação entre o crescente número de nascimentos de prematuros com o aumento dos casos dessa doença em todo mundo. Como exemplo, no Brasil, o número de prematuros em relação ao número total de nascimentos subiu de 3,72\% para $6,55 \%$, entre os anos de 1999 e 2005 , ou seja, praticamente dobrou em apenas seis anos (Figura I).

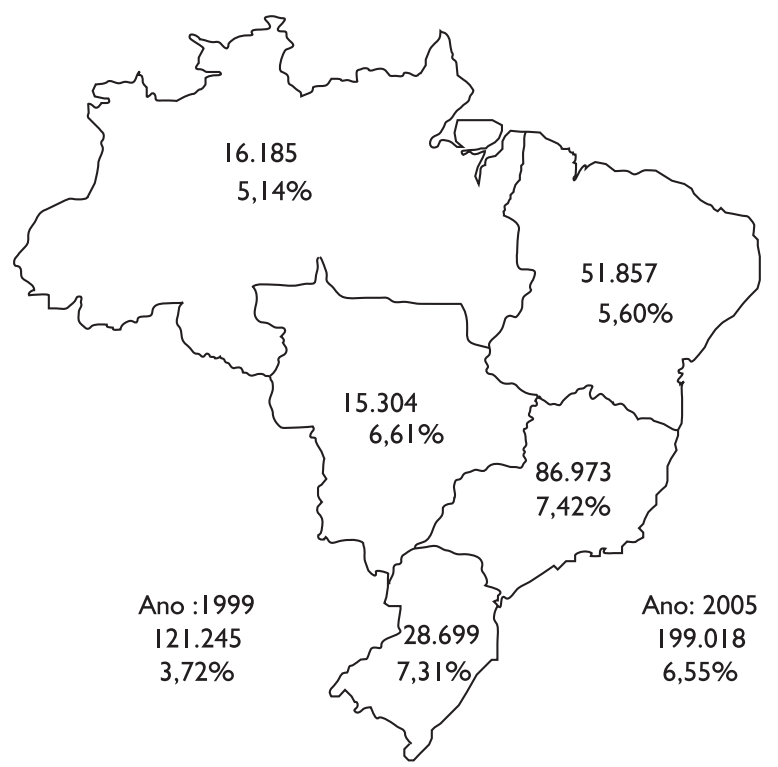

Figura I. Número e porcentagem relativa de recém-nascidos prematuros acometidos pela doença nas cinco regiões geográficas brasileiras nos anos de 1999 e 2005. ${ }^{(5)}$
Dessa forma, uma alternativa viável para aumentar a eficácia da fototerapia, e, consequentemente, eliminar possíveis problemas operacionais desse tratamento, é a utilização de sensores de acúmulo de dose de radiação para o monitoramento da radiação incidente em recém-nascidos ictéricos. ${ }^{(6-12)} \bigcirc$ uso desses selos permitiria a avaliação da equivalência das doses administrada e prescrita por pediatras para o controle da doença em cada neonato garantindo, assim, a segurança e a eficiência do tratamento.

Este trabalho tem como objetivo a caracterização e a fabricação de filmes finos baseados em materiais orgânicos luminescentes, usualmente empregados em dispositivos emissores de luz nanoestruturados: o tris (8-hydroxyquinolinato de alumínio) - $\mathrm{Alq}_{3}$ e o poli[2-metóxi-5-(2'etil-hexilóxi)-p-fenilenovinileno] - MEH-PPV, para uso como elemento ativo de sensores de acúmulo de dose de radiação azul.

\section{MATERIAIS E MÉTODOS}

Neste trabalho foram preparadas filmes de poli[2-metóxi-5-(2'-etilóxi)-p-fenilenovinileno] - MEH-PPV e Tris-(8-hidroxiquinolinolato) de alumínio (III) - $\mathrm{Alq}_{3}$ em matriz de poliestireno, todos obtidos da empresa Sigma Aldrich. O MEH-PPV foi escolhido por apresentar variação de suas propriedades ópticas quando exposto a radiação; já o $\mathrm{Alq}_{3}$, por apresentar sobreposição do seu espectro de emissão com o espectro de absorção do MEH-PPV, enquanto o PS pela sua transparência e baixo custo. Para fabricação os filmes foram preparadas soluções de MEH-PPV $(50 \mu \mathrm{g} / \mathrm{mL}), \mathrm{Alq}_{3}(100 \mu \mathrm{g} / \mathrm{mL})$ e PS (100 mg/mL) em clorofórmio, as quais foram depositadas em etiquetas de papel auto-adesivas via técnica casting. Uma vez preparados, os filmes foram expostos a diferentes doses de radiação azul proveniente um conjunto de LEDs azuis comumente utilizados no tratamento de icterícia em hospitais. A irradiação dos filmes foi realizada à temperatura ambiente e a $30 \mathrm{~cm}$ da fonte de luz para manter as mesmas condições do tratamento de icterícia $\left(40 \mu \mathrm{W} / \mathrm{cm}^{2} . \mathrm{nm}\right)$. Durante a irradiação, os filmes foram caracterizados por meio de medidas de fotoluminescência e de diagramas de cromaticidade com o espectrofotômetro USB2000 da marca Ocean Optics, uma vez que são excitados pela fonte de luz azul proveniente do equipamento fototerapêutico. É importante destacar que, neste trabalho, é usado tempo em vez de dose de radiação, uma vez que esse é o parâmetro usualmente utilizado nos tratamentos fototerápicos.

\section{RESULTADOS}

A Figura 2 mostra o espectro de fotoluminescência de um filme de PS/MEH-PPV/Alq ${ }_{3}$ exposto durante três horas à radiação. Observa-se nessa figura que 0 espectro de emissão do sistema se desloca para menores 
comprimentos de onda com o tempo de exposição $\left(t_{\mathrm{e}}\right)$ à radiação. De acordo com a literatura, ${ }^{(6-12)}$ há quebra da ligação vinílica $(C=C)$ e incorporação de carbonílas $(\mathrm{C}=\mathrm{O})$ na cadeia principal do MEH-PPV.

Dos resultados apresentados na Figura 2 observa-se, inicialmente, que o filme apresenta emissão máxima na região do vermelho-laranja $(\sim 600 \mathrm{~nm})$ que se desloca para o verde $(\sim 550 \mathrm{~nm})$ com o tempo de exposição à radiação. Esse resultado é relacionado a outros encontrados na literatura ${ }^{(10,12)}$, que discutem sobre os efeitos da radiação nos processos de degradação de soluções de MEH-PPV/Alq ${ }_{3}$ em clorofórmio. Estes mesmos resultados são apresentados na Figura 3 em gráficos do tipo diagrama de cromaticidade, que representam a percepção visual que um profissional da saúde teria ao analisar as alterações na fotoemissão dos filmes de MEH-PPV/Alq ${ }_{3} /$ PS sob exposição a fontes de luz azul. No gráfico apresentado na Figura 3 observa-se claramente a mudança de cor do sistema de vermelho-laranja ao verde com o tempo de exposição à radiação. Esta alteração de cor é usada para fabricação de selos autocolantes, cujo princípio de operação é descrito neste trabalho como a de um "semáforo inteligente", cuja mudança de cor do vermelho ao verde está diretamente relacionada à dose de radiação azul absorvida pelo material. Quando a mudança de cor for ajustada à dose de radiação prescrita para tratamento da doença, a cor verde indicará que o procedimento fototerápico foi realizado com sucesso.

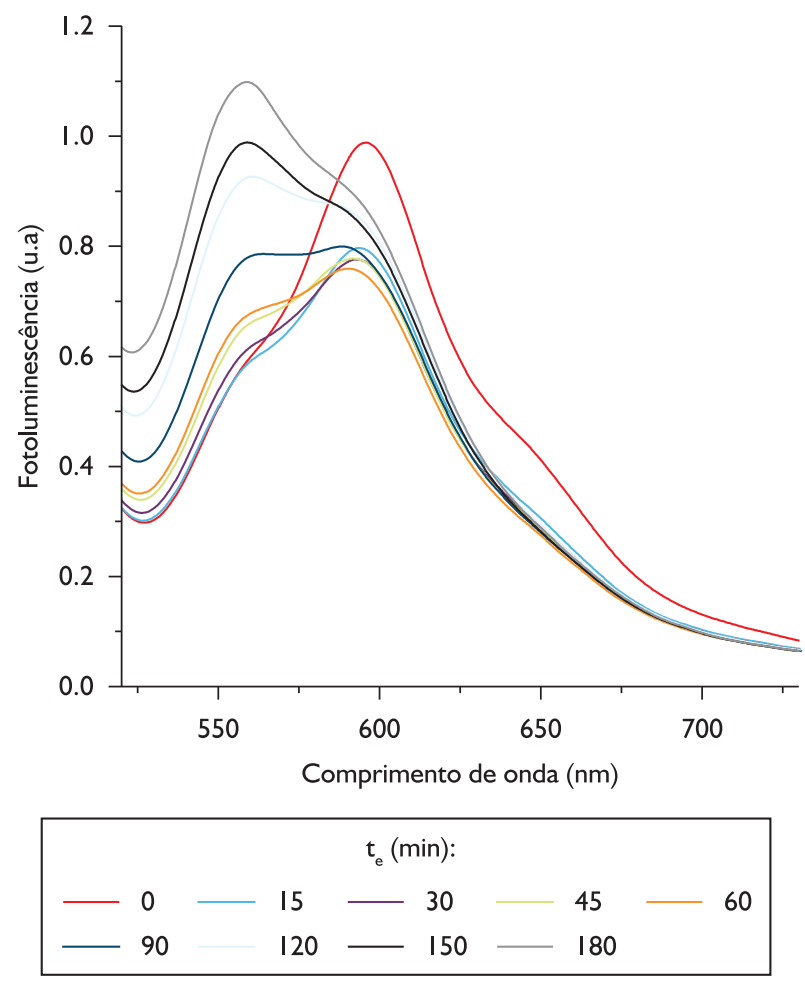

Figura 2. Espectro de fotoluminescência de filmes de PS/MEH-PPV/ $\mathrm{Alq}_{3}$ expostos à radiação durante três horas.

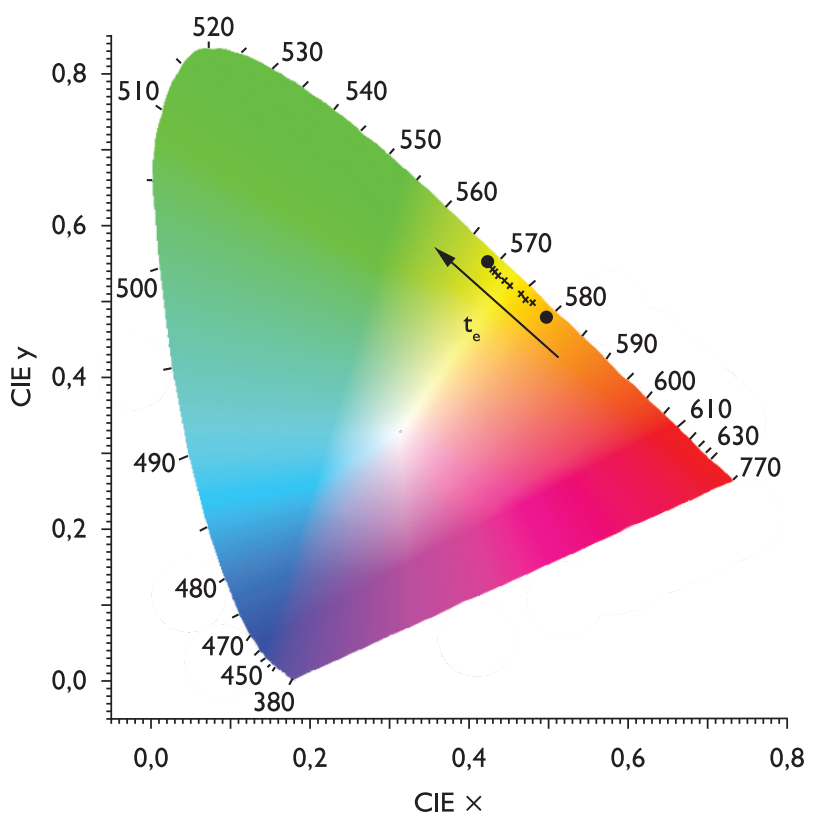

Figura 3. Diagrama de cromaticidade dos filmes de MEH-PPV/Alq $/$ PS expostos durante 180 minutos à radiação de luz azul.

A Figura 4 mostra uma sequência de filmes de MEH-PPV/Alq $q_{3} / P S$, cuja concentração de MEH-PPV usada para confecção desses sistemas foi modificada para $200 \mu \mathrm{g} / \mathrm{mL}$, expostos a 0, I h, $2 \mathrm{~h}, 3 \mathrm{~h}, 4 \mathrm{~h}, 5 \mathrm{~h}, 6 \mathrm{~h}, 7 \mathrm{~h}, 8 \mathrm{~h}$ $\mathrm{e} 9 \mathrm{~h}$ à radiação azul. $\mathrm{A}$ figura também mostra um boneco representando um neonato sob tratamento fototerápico cuja fralda apresenta ainda três selos de MEH-PPV/Alq ${ }_{3} /$ PS expostos a $0 \mathrm{~h}$ (vermelho), $4 \mathrm{~h}$ (laranja) e $9 \mathrm{~h}$ (verde) a radiação. Os resultados apresentados nessa figura demonstram não somente que a alteração na composição química das soluções usadas na fabricação dos selos pode alterar o tempo de alteração de cor dos sensores mas, sobretudo, que é possível confeccionar selos de forma amigável para uso em unidades de tratamento intensivo neonatal, e que, é possível ajustar e calibrar facilmente as curvas de resposta cor-dose dos sensores para a dose de radiação prescrita para $\circ$ tratamento da doença. Finalmente, a Figura 5 mostra fotos com cerca de 150 selos de $\mathrm{MEH}-\mathrm{PPV} / \mathrm{Alq}_{3} / \mathrm{PS}$ produzidos em escala piloto via método casting. $A$ foto foi obtida durante a exposição inicial dos selos a luz azul. A foto mostra algo em torno de I 50 selos que possuem como principais vantagens a facilidade de preparação e o baixo custo $(<\mathrm{R} \$ 0,50)$.

\section{DISCUSSÃO}

O uso de sistemas orgânicos luminescentes para aplicação como sensores de radiação azul já foi reportado na literatura ${ }^{(6-12)}$ pelos autores deste trabalho em artigos que tinham como objetivo principal a investigação do papel da radiação nas propriedades ópticas e estruturais 


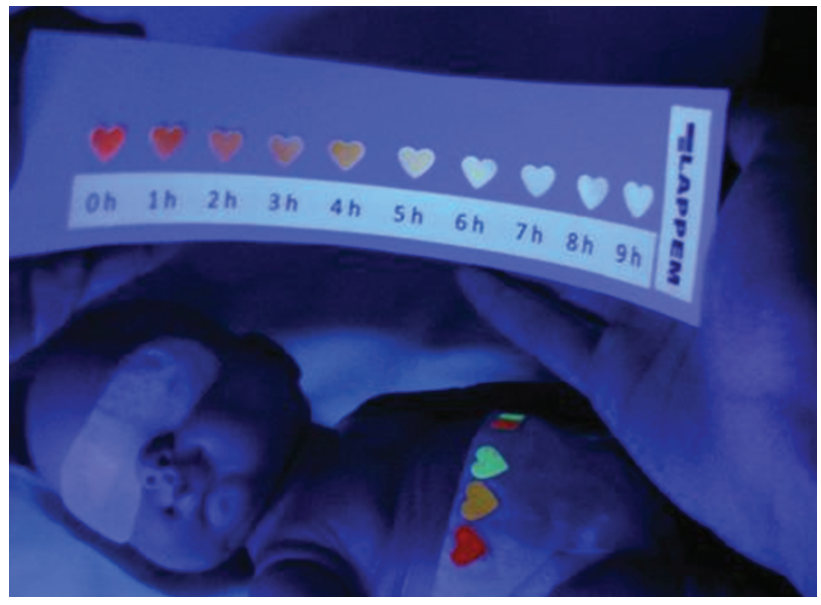

Figura 4. Filmes autocolantes de PS/MEH-PPV/Alq ${ }_{3}$ submetidos a diferentes tempos de exposição à radiação (de 0 a 9 horas).

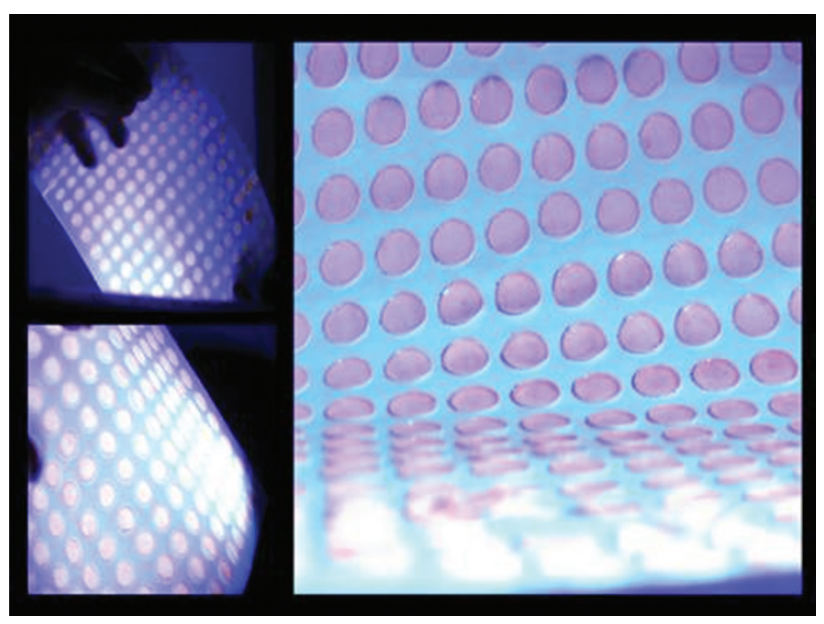

Figura 5. Sensores de radiação azul, baseados em $\mathrm{MEH}-\mathrm{PPV} / \mathrm{Alq}_{3} /$ OS, fabricados em escala piloto. A figura mostra a foto de cerca de I50 selos recém-fabricados excitados opticamente com luz azul. do MEH-PPV e do $\mathrm{Alq}_{3}$. Contudo, como a ênfase maior desses trabalhos foi a investigação fotofísica de soluções de MEH-PPV e de MEH-PPV/Alq $q_{3}$ em clorofórmio, pouco se contribui para a fabricação e otimização de sensores orgânicos eficientes para uso no controle das condições de iluminação de neonatos ictéricos sob tratamento fototerápico. Ademais, soluções em clorofórmio para uso em neonatologia são, sem dúvida, um problema de toxidade para a área médica e, portanto, um entrave médico-hospitalar para o uso desses sistemas. Nesse contexto, a fabricação de sensores de radiação na forma de selos autocolantes, que podem ser aderidos na fralda do recém-nascido evitando-se inclusive o contato direto com a sua pele, é a principal contribuição desse trabalho.

\section{CONCLUSÃO}

Os resultados apresentados neste trabalho mostram o potencial que filmes finos orgânicos luminescentes à base de MEH-PPV e $\mathrm{Alq}_{3}$, usualmente utilizados em dispositivos eletroluminescentes nanoestruturados, apresentam para uso como elemento ativo de sensores de radiação inteligentes para uso no monitoramento das condições de iluminação de recém-nascidos sob fototerapia neonatal. Outras vantagens desse dispositivo são: baixo custo, facilidade de fabricação e facilidade de leitura de dose, além da indicação direta da dose prescrita por pediatras para $\circ$ tratamento da doença por meio da simples comparação de cor garantindo, assim, a segurança e a eficiência do tratamento.

\section{Agradecimentos}

Apoio da REDENANOBIOMED/Capes, CNPq, INEO/CNPq, FAPITEC-SE/CNPq e Fapemig.

\section{REFERÊNCIAS}

I TAN, K. L. Phototherapy for neonatal jaundice. Acta Paediatrica, v. 85, n. 3, p. 277-9, Mar. 1996.

2 RAl, A. K. et al. Spectroscopic studies and normal coordinate analysis of Bilirubin. Spectrochimica Acta Part A, v. 58, n. 10, p. 2145-52, Ago. 2002.

3 TAN, K. L. The pattern of bilirubin response to phototherapy for neonatal hyperbilirubinaemia. Pediatric Research, v. 16, n. 8, p. 670-4, Ago. 1982.

4 IP, S. et al. Hyperbilirubinemia and kernicterus: 50 years later. Pediatrics, v. I I4, n. I, p. 263-4, Jul. 2004.

5 SEGATTO, C.; ANAUATE, G.; BUSCATO, M.. Bebês Prematuros. Revista Época, n. 53I, 21 jul. 2008. p. I04-I 2.

6 VASCONCELOS, C. K. B.; BIANCHI, R. F. Dosímetro de radiação não ionizante construído com polímeros conjugados. Patete $n^{\circ}$ PI 0700497-4 Brasil. 24 fev. 2007.

7 VASCONCELOS, C. K. B.; BIANCHI, R. F. Polímeros luminescentes como sensores de radiação não ionizante: aplicação em fototerapia neonatal. Polímeros: Ciência e Tecnologia, v. I7, n. 4, p. 325-8. Mar. 2007.

8 VASCONCELOS, C. K. B. et al. Polímeros luminescentes como sensores de radiação não ionizante: aplicação em fototerapia neonatal. In: BRASIL. Ministério da Saúde. Secretaria de Ciência, Tecnologia e Insumos Estratégicos. Departamento de Ciência e Tecnologia. Prêmio de Incentivo em Ciência e Tecnologia para o SUS: Edição 20 anos do SUS 2008. Brasília, DF, 2008. v. I, p. I24-8. 
9 FERREIRA, G. R. Preparação, caracterização e fabricação de sensores de acúmulo de dose de radiação azul baseado em sistemas orgânicos luminescentes. 2008. II 2 p. Dissertação (Mestrado em Engenharia de Materiais) - Instituto de Ciências Exatas e Biológicas da Universidade Federal de Ouro Preto, Ouro Preto, 2008.

I0 FERREIRA, G. R.; VASCONCELOS, C. K. B.; BIANCHI, R. F. Design and characterization of a novel indicator dosimeter for blue-light radiation. Medical Physics, v. 36, n. 2, p. 642-4, Jan. 2009.

II VASCONCELOS, C. K. B.; BIANCHI, R. F. A blue-light dosimeter which indicates the dose accumulation by a multicoloured change of photodegraded polymer. Sensors and Actuators. B, Chemical, v. I43, n. I, p. 30-34, Dec. 2009.

12 FERREIRA, G. R.; VASCONCELOS, C. K. B.; BIANCHI, R. F. Dosímetro pessoal de radiação azul baseado em sistemas orgânicos luminescentes.In: CONGRESSO BRASILEIRO DE FÍSICA MÉDICA, 14., 2009, São Paulo. Anais... São Paulo: Anvisa, 2009. p. I-4.

Recebido em: 03/I2/2010

Aceito em: 30/05/20I I 